\title{
Investigation of VEGF and PDGF signals in vascular formation by 3D culture models using mouse ES cells
}

\author{
Hitomi Hosoe ${ }^{1}$, Yuri Yamamoto ${ }^{1}$, Yusuke Tanaka ${ }^{1}$, Mami Kobayashi ${ }^{2}$, Nana Ninagawa ${ }^{2}$, \\ Shigeko Torihashi, ${ }^{1,2^{*}}$ \\ ${ }^{1}$ Department of Physical Therapy, Nagoya University School of Health Sciences, Nagoya, Japan; \\ *Corresponding Author: storiha@met.nagoya-u.ac.jp \\ ${ }^{2}$ Department of Health Sciences, Nagoya University Graduate School of Medicine, Nagoya, Japan
}

Received 13 February 2012; revised 18 March 2012; accepted 14 April 2012

\begin{abstract}
Vascular formation in vivo involves several processes and signal cascades subsequently occurring in the embryo. Several models by ES cells have been reported for analysis in vitro. We show here a 3D culture system using collagen gel (AteloCell) as a simple and useful system for investigating vascular formations and analyzing the roles of factors in vivo. Although VEGF and PDGF are growth factors with multi-potentials for vascular formation, their sequential roles have not been elucidated. We investigated the effects of VEGF and PDGF $B$ signals for vascular formation by a 3D culture system that embedded embryoid bodies (EBs) from ES cells into a collagen gel. After embedding EBs in the collagen gel with a medium containing VEGF, EBs gave off CD105 immunopositive vessels as the initial step of vasculogenesis. When the factor in the culture medium for EBs was switched from VEGF to PDGF $B$ after 5 days of culture, the morphological features of vessels varied, suggesting the occurrence of vascular-type differentiation. After 11 days of 3D culture, vessels in both groups cultured with VEGF alone and switching to VEGF $B$ at day 5 showed Flk-1 immunoreactivity. Some blood vessels cultured with PDGF B after day 5 expressed either EphrinB2 (arteriole marker) or Flt-4 (lymphatic marker) immunoreactivity, but vessels cultured with VEGF alone exhibited neither of them. Vessels cultured with these two factors could not differentiate into a venous type. The present study indicates that VEGF is the initial signal for vasculogenesis, and that PDGF $B$ is probably involved in vascular diversification.
\end{abstract}

Keywords: Vasculogenesis; Angiogenesis; VEGF;
PDGF; ES Cells; 3D Culture Model; Collagen Gel

\section{INTRODUCTION}

ES cells with pluripotency have been used to investigate the vascular formations involving primitive blood vessel formation, the differentiation of endothelial cells with a lumen, the assembly into cord-like structure, and the differentiation of vascular types. Early investigations in vitro used embryoid bodies (EBs) that resembled developing embryos in a 2D culture system [1]. Instead of 2D growths of vessels, 3D cultures showed a further similarity to vasculogenesis in vivo. Although Matrigel provide a useful material for 3D culture, several unqualified inclusions have interfered with a critical analysis [2-4]. The AteloCell, a highly purified and cutting off antigenic region of type I collagen proved more suitable than Matrigel for a 3D culture, and has been commonly used recently [5-7]. We used AteloCell collagen gel and embedded EBs completely into it in our model. Therefore, all blood vessels protruding from EBs expanded 3 dimensionally, similar to those in the embryo. Because the initiation of vasculogenesis involves several signal cascades and processes subsequently occur in the embryo, it still remained unclear and is worthy of investigation using a $3 \mathrm{D}$ culture system in vitro.

VEGF is well known as a key factor for both vasculogenesis as well as angiogenesis [8-11]. PDGF B is also a critical factor for the development of blood vessels $[12,13]$. Both factors independently participate in several steps of vascular formation. However, sequential usage of VEGF and PDGF B promoted angiogenesis in the myocardium following infarction [14]. Therefore, a combination of those two growth factors would produce non prospective effects on the blood vessel development that actually occurs in vascular formation in vivo. In the present study, we employed a 3D culture model using collagen gel, and analyzed the subsequent participation of two factors i.e., VEGF and PDGF B in a vascular forma- 
tion. Our findings indicate that VEGF initiated vasculogenesis, and PDGF B may have been involved in the differentiation of vascular types.

\section{MATERIALS AND METHODS}

\subsection{Culture of Mouse ES Cells (M-ESCs)}

Mouse ES cells (M-ESCs: G4-2; carrying the enhanced green fluorescent protein-EGFP-gene under the control of cytomegalovirus/chicken $\beta$-actin promoter) were expanded in the culture medium termed ES-DMEM. That is, Dulbecco's modified Eagle's medium (Sigma; St. Louis, MO; www.sigma-aldrich.com) with $0.1 \mathrm{mM}$ non-essential amino acids (GIBCO; Carlsbad, CA; www.invitrogen.com), $100 \mathrm{mM}$ sodium pyruvate (GIB$\mathrm{CO}$ ), $100 \mathrm{mM}$ 2-mercaptoethanol (Sigma), and 0.5\% antibiotic-antimycotic (GIBCO) containing 10\% fetal bovine serum (FBS; Biological Industries, Kibbuiz, Israel; www.bioind.com). For the expansion of M-ESCs, $1,000 \mathrm{U} / \mathrm{mL}$ of leukemia inhibitory factor (LIF; Chemicon, Temecula, CA; www.millipore.com) was added to ES-DMEM.

\subsection{Induction to Mesodermal Cells and Preparation of 3D Culture}

ES cells were changed to ES-DMEM (without LIF) to form embryoid bodies (EBs). About 660 cells in each well of a Micro Sphere array (MSCW1-CA600, STEM Biomethod http://stem-biomethod.co.jp) were compacted to form EBs for 2 days and treated with $10-3 \mathrm{mM}$ all-trans retinoic acid (RA: R2625, Sigma) for 1 day. After 2 days of rinsing by ES-DMEM, EBs were embedded into collagen gel (AteloCell, DME-02; KOKEN Tokyo, www.kokenmpc.co.jp). About 30 EBs in each 35mm dish (BD Falcon; www.bd.com) were mounted in 1 $\mathrm{mL}$ of collagen gel and incubated at $37^{\circ} \mathrm{C}$ for one hour. After the solidifying of collagen gel, $1.5 \mathrm{~mL}$ of culture medium was added. At that moment, the date of 3D cul- ture was designated as Day 0.

\subsection{Medium Condition}

From days 0 to 4, some EBs termed VEGF+ were incubated in ES-DMEM with $50 \mathrm{ng} / \mathrm{mL}$ of VEGF (VEGF A; mBA-165, Santa Cruz Biotech, Santa Cruz, CA.; www.scbt.com). As a control, EBs cultured with ESDMEM alone were termed VEGF-. After day 5, EBs switched the medium from ES-DMEM with VEGF to ES-DMEM with $50 \mathrm{ng} / \mathrm{mL}$ of PDGF B (PDGF-BB, 100 14B PeproTech. Rocky Hill NJ.; www.peprotech.com) and were designated a PDGF B group (Figure 1). After day 5 , EBs that continued to be cultured with VEGF were subsequently termed VEGF+. All culture samples were incubated at $37^{\circ} \mathrm{C}$ with $5 \% \mathrm{CO} 2$, and the medium was changed every other day.

\subsection{Fluorescent Immunostaining}

Samples in 3D culture were fixed with $4 \%$ paraformaldehyde in PBS for 10 minutes. After washing with PBS, the samples were treated with blocking solution containing $10 \%$ normal goat or donkey serum and $0.3 \%$ triton X-100 in PBS for 15 minutes. Some samples were frozen and sectioned ( $6 \mu \mathrm{m}$ thickness) by a Cryostat (CM1510, Leica; Nussloch Germany; www.hbu.de). Samples and sections were incubated with the first antibody followed by a brief rinse with PBS and were treated with Alexa-labeled secondary antibody. The antibodies used are listed in Table 1, and first antibodies were incubated at $4{ }^{\circ} \mathrm{C}$ for 12 hours. The secondary antibodies were diluted 400 times and used for $30 \mathrm{~min}$. They were rinsed with PBS, then stained with DAPI (1:1000, KPL Gaithersburg MD; www.kpl.com) for 3 min followed by a mounting with FluoromountTM (DBS, Pleasanton, CA; www.dibisys.com). Samples were observed using a fluorescent microscope, BZ-9000 (Keyence Osaka Japan; www.keyence.co.jp). Statistical analysis was carried out using a BZ-II analyzer (Keyence).

Table 1. Antibodies for fluorescent immunostaining.

\begin{tabular}{lllc}
\hline \multicolumn{1}{c}{ Antibody (IgG) } & \multicolumn{1}{c}{ Origin } & Dilution \\
\hline PECAM-1 (Goat) & sc-1506 & Santa Cruz Biotech & $1: 50$ \\
EphrinB2 (Goat) & AF496 & R\&D system; Minneapolis MN; www.RnDSystems.com & $1: 50$ \\
Flt-4 (Rabbit) & 53493 & AnaSpec; San Jose. CA; www.anaspec.com & $1: 100$ \\
Flk-1 (Rabbit) & ab39256 & abcam; Tokyo Japan; www.abcam.co.jp & $1: 100$ \\
PDGFR- $\beta$ (Rabbit) & Ab-1 & Thermo; Fremount CA; www.thermo.com & $1: 200$ \\
Eph receptor B4 (Rat) & ab73259 & abcam & $1: 100$ \\
PROX1 (Mouse) & M05 & Abmova; Taipei Taiwan; www.abnova.com.tw & $1: 80$ \\
CD105 (Rat) & MAB1320 & R\&D & $1: 100$ \\
\hline
\end{tabular}

Secondary antibodies: Alexa Fluor 594 donkey anti-goat IgG (A11058. Mo- lecular Probes, Leiden, Netherlands; www.probes.com), Alexa Fluor 594 goat anti-rabbit IgG (A11012), Alexa Fluor 594 goat anti-rat IgG (A11007), Alexa Fluor 594 goat anti-mouse IgG (A11005) and Alexa Fluor 488 donkey anti(A11006), Alexa Fluor 350 goat anti-rat IgG (A21093). 


\subsection{Morphological Analysis}

EBs or vessels (budding) from VEGF+, VEGF-, and PDGF B groups were randomly chosen. From days 1 to 4 , the number of EBs with budding was counted with three $35 \mathrm{~mm}$ culture dishes in each group. The length of 10 buddings was measured every 5 EBs in three dishes. On day 11, the length, thickness and number of branches of 10 vessels for every $5 \mathrm{EBs}$ in three dishes were counted. Vessel thickness was measured at the mid-portion of the vessels from EB to their tips. Student $t$-tests were used to examine the results. Data are presented as mean $\pm \mathrm{SD}$. A significance level of $p<0.05$ was chosen.

\subsection{Gene Expression Profiling Using Real-Time PCR}

Functional genes were analyzed using a mouse angiogenesis RT2 ProlilerTM PCR array (PAMM-024A; SA Bioscience, Frederick, MD, www.SABiosciences.com). EBs at day 0 and samples of either VEGF+ or PDGF B at day 11 were collected from 3D culture dishes (Figure 1). EBs and protruding vessels with small amounts of surrounding collagen gel were taken from the dishes and crushed using an ultrasound-sonic pulverizer for $30 \mathrm{sec}-$ ond at $4^{\circ} \mathrm{C}$ (Handy sonic; Tomy Seiko Tokyo,

http://bio.tomys.co.jp). Subsequently, total mRNAs from these samples were extracted by an RNeasy micro kit (Qiagen, Valencia, CA; www.quiagen.com) according to the manufacturer's instructions. RNAs were immediately reverse-transcripted to cDNA by SuperScriptTM II (Qiagen). Using real-time PCR, 84 genes involved in modulating the biological processes of angiogenesis were analyzed following the manufactures instructions. Sample cDNAs were poured into 96-well plates and pro-

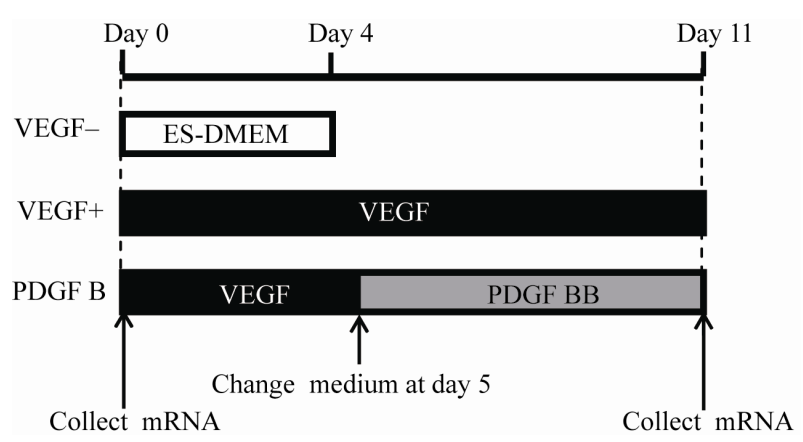

Figure 1. Schema of experimental groups and time schedule. The day when embryoid bodies (EBs) were embedded into AteloCell collagen gel as 3D culture was designated as Day 0. VEGF- group was cultured in the ES-DMEM for 4 days. VEGF+ group was incubated in the medium containing VEGF until day 11. PDGF B group was incubated with the medium containing VEGF that was switched to new one including PDGF B instead of VEGF at day 5. For PCR array, mRNAs were collected from EBs on day 0 and from samples of VEGF+ and PDGF B on day 11. cessed using a personal real-time PCR system (Mx3000p Stratagene; La Jolla. CA: www.stratagene.com). PCR was amplified to 40 cycles at $95^{\circ} \mathrm{C}$ for 15 seconds and $60^{\circ} \mathrm{C}$ for 60 seconds.

\section{RESULTS}

\subsection{First Step of Vasculogenesis by VEGF}

At day 1, some EBs sprouted a small number of short buds. The number of EBs with budding was higher in VEGF+ than that of VEGF-. On day 2, VEGF+ increased the EBs with budding, and the number of those EBs was remarkably higher than those of VEGF-. However, on days 3 and 4, VEGF- rapidly increased those EBs with processes growing from their buds. The difference in the number of EBs with budding between VEGF+ and VEGF- became smaller. Although the length of processes budding from EBs in both VEGF+ and VEGF- increased, it was longer in VEGF+ than that in VEGF- (Figure 2). To determine whether or not processes in VEGF+ and VEGF- were blood vessels, the expression of blood vessel marker proteins was examined by immuno-histochemistry. EBs at day 0 expressed Flt-1 (VEGF receptor 1) but not PDGF receptor $\beta$ (data not shown). The processes of VEGF+ exhibited CD105 immunoreactivity by day 4 , but after longer incubation they all showed both CD105 and PECAM 1 by day 11 (Figure 3). On the other hand, processes of VEGF- on day 4 expressed CD105, but did not show PECAM1 by day 11 (data not shown).

\subsection{Effects of PDGF $B$}

At day 5, half of the dishes embedding EBs were switched from a medium with VEGF to one containing PDGF B, and were termed the PDGF B group (Figure 1). After that, EBs of both VEGF+ and PDGF B groups increased blood vessels gradually (Figures 4(a)-(d)). At day 11, morphological features of the vessels differed between the two groups. Vessels of VEGF+ became longer and their average number of branches was higher than those of the PDGF B. Vessels of VEGF+ were thin and branched frequently to make a network or meshwork. The distribution area of vessels was more extensive than that of the PDGF B group (Figures 4(e)-(f)). On the other hand, many vessels of PDGF $B$ thickened, and were shorter in the length than those in VEGF+ (Figure 4(g)). PDGF B altered the morphological features of the vessels, suggesting that it induced a differentiation of vessel types.

\subsection{Expression of Vessel-Type Markers}

Expression of marker proteins was examined by immuno-histochemistry on day 11 to identify the type of 


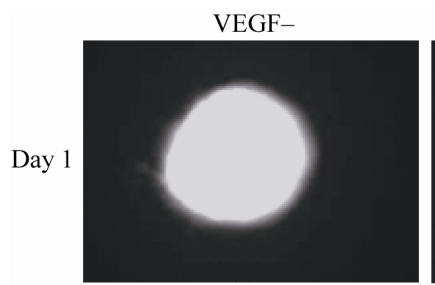

(a)

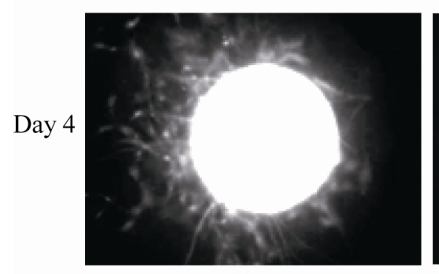

(c)

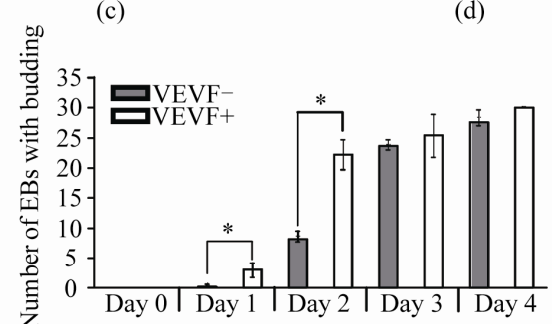

(e)

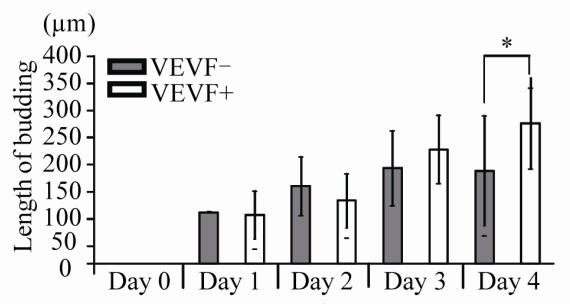

(f)

Figure 2. EBs with budding processes and their morphological alteration in VEGF- and VEGF+ groups EBs with budding processes in VEGF- and VEGF+ were demonstrated by EGFP fluorescent on day $1((\mathrm{a}),(\mathrm{b}))$ and day 4 ((c), (d)), and their numerical data were summarized in graphs $\mathrm{E}$ and $\mathrm{F}$. The total number of EBs with processes was counted in three $35 \mathrm{~mm}$ dishes of VEGF+ and VEGF- groups (e). The length of the processes was measured in 10 processes of every $5 \mathrm{EBs}$ in three dishes randomly chosen (f). Scale bar $(100 \mu \mathrm{m})$ is common in (a)-(d). Data are presented as mean \pm SD. ${ }^{*} \mathrm{p}<0.05$.
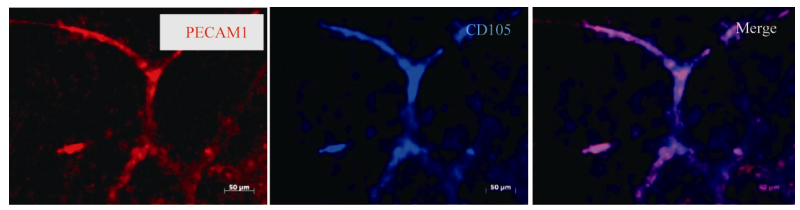

Figure 3. Expression of blood vessel markers. The processes of VEGF+ showed both CD105 and PECAM 1 (endothelial markers) immunoreactivity on day 11 . Scale bar is $50 \mu \mathrm{m}$.

vessels. All vessels expressed PECAM 1 in both the VDGF+ and PDGF B groups. Vessels in the PDGF B group exhibited either Ephrin-B2, Flt-4 or PROX1 immunoreactivity, whereas none of $\mathrm{VEGF}+$ expressed them (Figures 5(a)-(c)). Ephb4, a marker of veins, was ex-

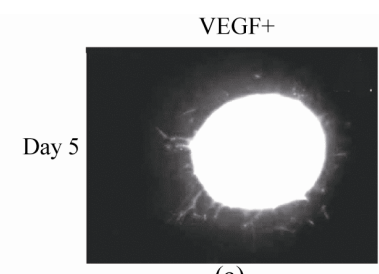

(a)

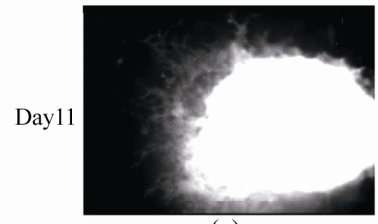

(c)
PDGF B

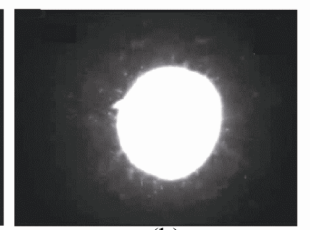

(b)

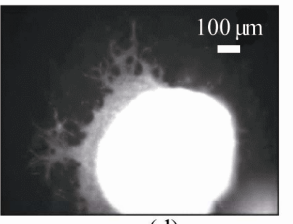

(d)

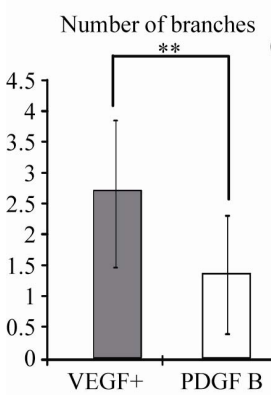

(e)

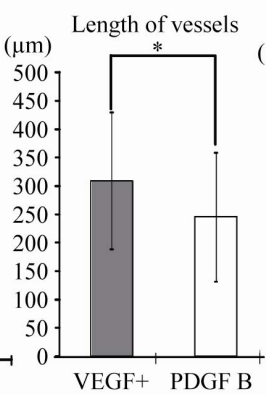

(f)

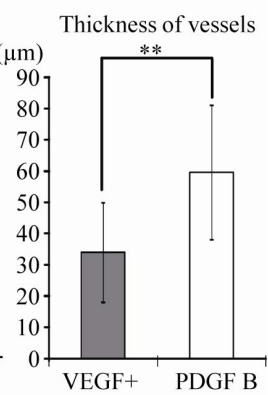

(g)
Figure 4. Morphological feature of vessels in VEGF+ and PDGF B. EBs and vessels in VEGF+ and PDGF B were demonstrated by EGFP fluorescent on day $5(\mathrm{a}, \mathrm{b})$ and day $11(\mathrm{c}, \mathrm{d})$, and their numerical data were summarized in graphs $\mathrm{E}, \mathrm{F}$ and $\mathrm{G}$. On day 11, the number of branches, length and thickness of vessels were counted on 10 vessels for every $5 \mathrm{EBs}$ in three dishes of VEGF+ and PDGF B groups respectively. Scale bar $(100 \mu \mathrm{m})$ is common in A-D. Data are presented as mean $\pm \mathrm{SD}$. p $<0.05,{ }^{* *} \mathrm{p}<0.01$.

pressed in neither the VEGF+ nor PDGF B groups (data not shown). Blood vessels in both groups were Flk-1immunopositive. In the cross sections, Flk 1-positive endothelium formed tubular structures with a lumen at the center of the epithelium. Not all but some of the Flk-1 positive epithelium also showed CD105 (Figure 5(d)).

\subsection{Expression of mRNAs in Vessels}

Expression profiles of mRNA in vessels of the VEGF+ and PDGF B groups were also analyzed by PCR array. A total of 84 genes involved in angiogenic factors that included growth factors and receptors, adhesion molecules, proteases, their inhibitors, matrix proteins, transcription factors and others were compared to both groups of vessels, and were calibrated with mRNA from EBs on day 0 . Quite different expression profiles are summarized in Table 2. Important genes are shown in Figure 6(a). As shown by the immuno-histochemistry, Ephb4 was low in both VEGF+ and PDGF B supporting protein levels. $P d g f a$ was expressed at a very low level in both vessels. 


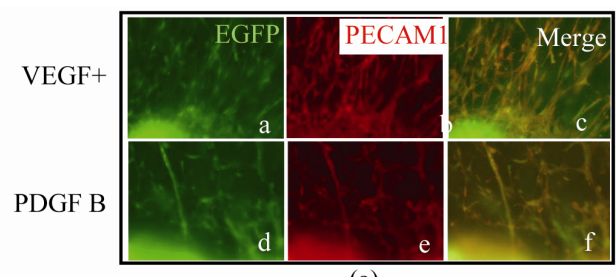

(a)
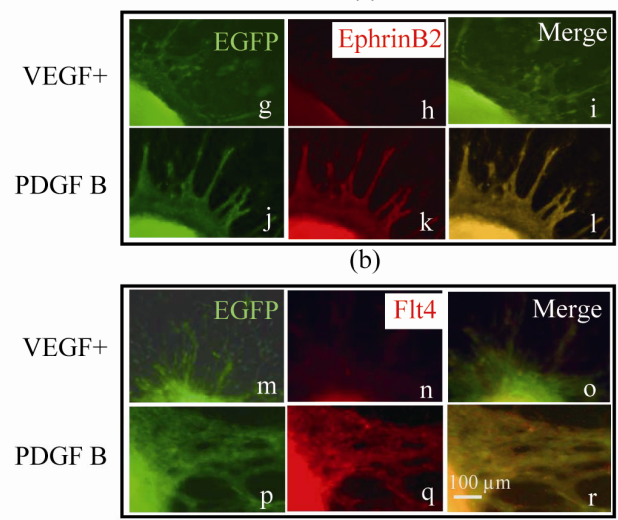

(c)

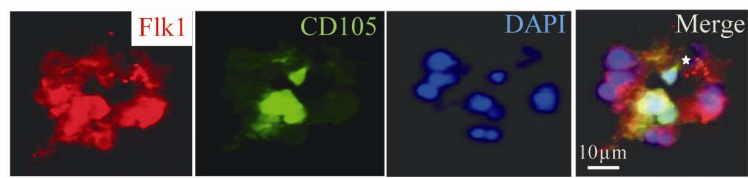

(d)

Figure 5. Immunofluorescent analysis of vessel types and existence of the lumen in the vessel at day 11. Common marker of blood vessels, PECAM1 was expressed in both VEGF+ and PDGF $B$ as shown in (a) (a - f). In panel (b), arterial marker ephrin B2 was expressed in PDGF B $(\mathrm{j}-1)$, but not in VEGF+ $(g-i)$. In panel (c), Lymphatic vessel marker flt4 was expressed in PDGF B $(p-r)$, but not in VEGF+ $(m-o)$. Scale bar $(100$ $\mu \mathrm{m})$ is common in (a)-(c). In the cross section of blood vessels in (d), flk1 immunopositive endothelial cells surround the lumen (asterisk) and some endothelium also showed CD105 immunoreactivity.

Although VEGF was not added to the media of PDGF B after day 5, the mRNA of Vegf $a$ was higher in BDGF B than that of $\mathrm{VEGF}+$. Several mRNAs showed contradictory expression patterns. The number of mRNAs that increased expression intensity from EBs at day 0 was higher in PDGF B than that of VEGF+ (Figure 6(b)). Furthermore, the increases of the mRNA in PDGF B were more prominent than those of VEGF+. The word "data" is plural, not singular.

\section{DISCUSSION}

\subsection{Three-Dimensional Culture in Collagen Gel}

Our 3D culture system presents an environment more analogous to vascular formation in vivo than $2 \mathrm{D}$ culture. EBs were embedded in a collagen gel that avoided inclusion of the unknown materials involved in Matrigel.
Table 2. Genes showed considerable different expressions.

\begin{tabular}{ccc}
\hline gene & VEGF + & PDGF B \\
\hline Angpt1 & -1.10 & 4.14 \\
Bai1 & 9.46 & 26.69 \\
Ccl2 & 2.52 & 8.99 \\
Tymp & 7.96 & 3.16 \\
Epas1 & 5.95 & 10.84 \\
Flt1 & 9.08 & 3.86 \\
Fzd5 & 11.17 & 3.50 \\
Hand2 & 5.59 & 1.37 \\
Itgav & 1.68 & 5.73 \\
Npr1 & -2.28 & 1.86 \\
Plg & 13.29 & 4.16 \\
Tbx1 & 6.07 & 1.60 \\
Tbx4 & 3.37 & 13.91 \\
Tgfb2 & 2.40 & 7.05 \\
Tgfb3 & 1.66 & 8.68 \\
Tgfbr1 & -1.17 & 5.06 \\
Thbs1 & -1.53 & 3.31 \\
Vegfa & 7.79 & 12.54 \\
\hline
\end{tabular}

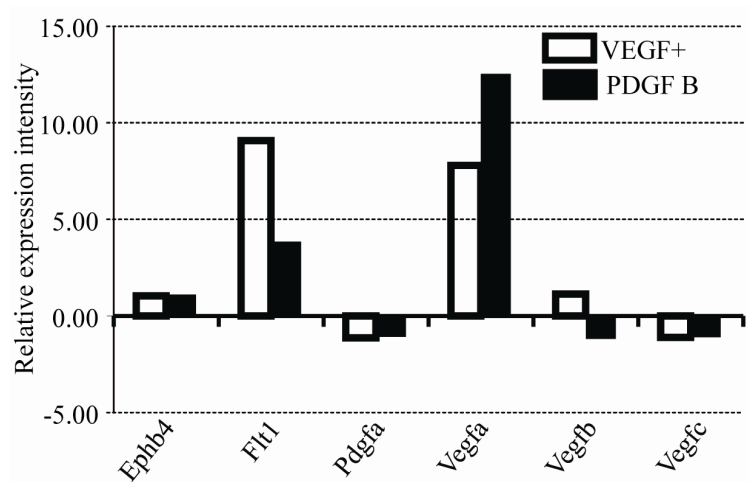

(a)

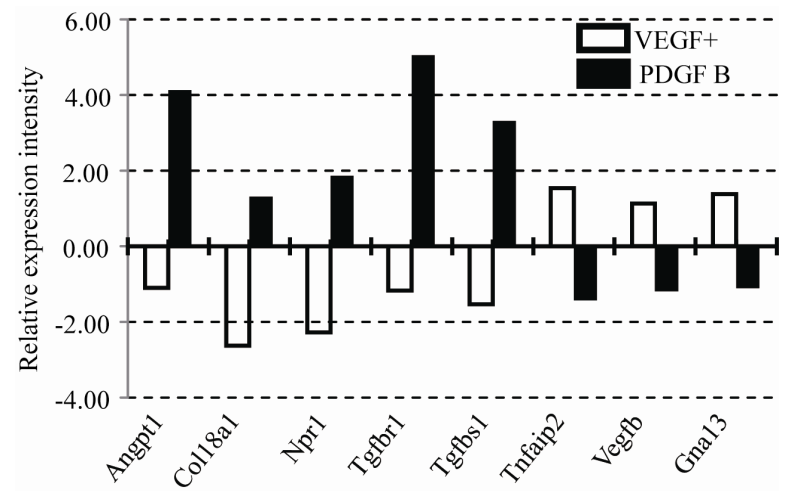

(b)

Figure 6. Expression profile of mRNAs in VEGF and PDGF B. Relative expression intensities of mRNAs in VEGF+ and PDGF $\mathrm{B}$ are summarized in two graphs (a) and (b). The value of EBs on day 0 is used as a calibrator and indicated as 1.00 . Important genes for vessel types and factors are shown in (a). Contradictory expression pattern is shown in (b). 
Therefore, we were able to simply compare the potency of two factors, VEGF and BDGF B. Before embedding into collagen gel, ES cells were treated for 2 days with RA during the formation of EBs. This short treatment with RA is important to induce cells into mesodermal lineage expressing VEGF receptor, Flk-1 [15]. Longer treatment with RA leads to a neuronal lineage (data not shown) [16].

\subsection{VEGF Initiates Vasculogenesis}

When EBs were cultured with VEGF (VEGF+), they protruded processes earlier than EBs without VEGF (VEGF-). Although EBs in VEGF- sprouted processes slightly later than $\mathrm{VEGF}+$, the number of their processes showed a similarity to those of VEGF+ at day 4. The processes from both groups of EBs expressed CD105 immunoreactivities at day 4, while VEGF+ also expressed PECAM1 at day 11. These observations confirmed that the processes induced by VEGF were blood vessels. A previous report by Seifert et al. described VEGF as having no effect on ES cells in the differentiation of endothelial cells of blood vessels [17]. They added VEGF (30 ng/mL) directly to ES cells when they began to differentiate due to the withdrawal of LIF. Their ES cells had never been pretreated with RA. Thus, VEGF as shown here was able to initiate vasculogenesis on the mesodermal lineage cells by the treatment with RA. EBs in VEGF- protruded processes slightly later than VEGF+. EBs induced to mesodermal cells by RA that could produce VEGF by themselves, and provided it endogenously for vasculogenesis[7,13]. Perhaps EBs in VEGF- were initiated to vasculogenesis by a small amount of VEGF originally involved in ES-DEME [5,18].

\subsection{Vascular Diversification by PDGF B Signal}

PDGF $\mathrm{B}$ has been described as a multiple player in vasculogenesis and angiogenesis. It works on the maturation of blood vessels to develop mural cells/pericytes, angiogenic sprouting, and an increase in VEGF signals through VEGF A; VEGF receptor 2 [6,7,12,13]. However, an effective role for the differentiation of vessel types has not been well investigated.

Our present data indicate that PDGF B rather than VEGF induced the differentiation of vessels. After switching the medium at day 5, the shape of vessels was altered in PDGF B, when the vessels became thick, short and less branched. In fact, Angpt 1 (Angiopoietin 1) mRNA which regulates the blood vessel caliber was extremely up-regulated in PDGF B (Figure 6(b)). The markers of vessel types were revealed by immunostaining. Although the vessels in both VEGF+ and PDGF B expressed PECAM 1 (a blood vessel marker), only ves- sels in PDGF B showed immunoreactivities of either Eph B2 (an arterial marker) or Flt4 (a lymphatic marker). That was also confirmed by an mRNA expression profile by a PCR array (Table 2). A variety of mRNAs increased their expression in PDGF B (Figure 6(b)). Although VEGF had never been added to the culture medium of the PDGF B group, the mRNA of Vegf $a$ was higher in the PDGF B group than that of VEGF+. After the activation of vasculogenesis by VEGF, vessels in PDGF $B$ were able to produce VEGF on their own $[7,13]$. On the other hand, VEGF failed to induce the generation of PDGF B by day 11 . Our results imply that the differentiation of vessel types requires a PDGF $B$ signal in addition to VEGF. Cao et al. described PDGF B-induced lymphangiogenesis that had the potential to grow lymphatic vessels in their system using mouse cornea [19]. PDGF B stimulated both MAP kinase activity and cell motility [12]. Our morphological data support their findings that PDGF $\mathrm{B}$ induced the differentiation of lymphatic vessels, though their underlying molecular mechanisms have not been clarified.

Arterial-venous specifications have not been fully elucidated. Recently, notch and $\beta$-catenin signaling, in addition to VEGF, have been postulated an effective for arterial specifications [20]. Yamamizu et al. and others have described cAMP as also being involved in the arterial fate. When such signals are blocked, the arterial fate alters to a venous one under the influence of VEGF, which basically induces venous characteristics in vascular progenitors [21-23]. Our findings have been somewhat controversial. Neither VEGF+ nor PDGF B induced venous differentiation. Probably an unknown signal except for VEGF will be required in our system to differentiate into venous vessels. In the present study, PDGF B showed the potential to differentiate into arterial and lymphatic type vessels. As shown in Table 2, PDGF B increased the TGF- $\beta$ family that might be implicated in such vascular specification [24,25]. Further investigation is warranted.

In summary, our 3D culture system using mouse ES cells and collagen gel is both simple and useful, and provides an analytical model for investigating vascular formation. The present study indicates that VEGF is the initial signal for vasculogenesis, and PDGF B is probably involved in vascular diversification.

\section{ACKNOWLEDGEMENTS}

We wish to thank Dr. H. Niwa (RIKEN, Kobe Japan) for his generous gift of mouse ES cells. This work was supported by Grants-in-Aid for Scientific Research Japan (S) and (B).

\section{REFERENCES}

[1] Wang, Z., Cohen, K., Shao, Y., Mole, P., Dombkowski, D. 
and Scadden, D.T. (2004) Ephrin receptor, EphB4, regulates ES cell differentiation of primitive mammalian hemangioblasts, blood, cardiomyocytes, and blood vessels. Blood, 103, 100-109. doi:10.1182/blood-2003-04-1063

[2] Gerecht-Nir, S., Ziskind, A., Cohen, S. and ItskovitzEldor, J. (2003). Human embryonic stem cells as an in vitro model for human vascular development and the induction of vascular differentiation. Laboratory Investigation, 83, 1811-1120. doi:10.1097/01.LAB.0000106502.41391.F0

[3] Nakagami, H., Nakagawa, N., Takeya, Y., Kashiwagi, K., Ishida, C., Hayashi, S., Aoki, M., Matsumoto, K., Nakamura, T., Ogihara, T. and Morishita, R. (2006) Model of vasculogenesis from embryonic stem cells for vascular research and regenerative medicine. Hypertension, $\mathbf{4 8}$, 112-119. doi:10.1161/01.HYP.0000225426.12101.15

[4] Boyd, N.L., Dhara, S.K., Rekaya, R., Godbey, E.A., Hasneen, K., Rao, R.R., West III, F.D., Gerwe, B.A. and Stice, S.L. (2007) BMP4 promotes formation of primitive vascular networks in human embryonic stem cellderived embryoid bodies. Experimental Biology and Medicine (Maywood), 232, 833-843.

[5] Feraud, O., Cao, Y. and Vittet, D. (2001) Embryonic stem cell-derived embryoid bodies development in collagen gels recapitulates sprouting angiogenesis. Laboratory Investigation, $\mathbf{8 1}, 1669-1681$.

doi:10.1038/labinvest. 3780380

[6] Lindskog, H., Athley, E., Larsson, E., Lundin, S., Hellström, M. and Lindahl, P. (2006) New insights to vascular smooth muscle cell and pericyte differentiation of mouse embryonic stem cells in vitro. Arteriosclerosis, Thrombosis, and Vascular Biology, 26, 1457-1464. doi:10.1161/01.ATV.0000222925.49817.17

[7] Magnusson, P.U., Looman, C., Ahgren, A., Wu, Y., Claesson-Welsh, L. and Heuchel, R.L. (2007) Platelet-Derived growth factor receptor-beta constitutive activity promotes angiogenesis in vivo and in vitro. Arteriosclerosis, Thrombosis, and Vascular Biology, 27, 2142-2149. doi:10.1161/01.ATV.0000282198.60701.94

[8] Yurugi-Kobayashi, T., Itoh, H., Yamashita, J., Yamahara, K., Hirai, H., Kobayashi, T., Ogawa, M., Nishikawa, S., Nishikawa, S.-I. and Nakao, K. (2003). Effective contribution of transplanted vascular progenitor cells derived from embryonic stem cells to adult neovascularization in proper differentiation stage. Blood, 101, 2675-2678. doi:10.1182/blood-2002-06-1877

[9] Coultas, L., Chawengsaksophak, K. and Rossant, J. (2005) Endothelial cells and VEGF in vascular development. $\mathrm{Na}$ ture, 438, 937-945.

[10] Lanner, F., Sohl, M. and Farnebo, F. (2007) Functional arterial and venous fate is determined by graded VEGF signaling and notch status during embryonic stem cell differentiation. Arteriosclerosis, Thrombosis, and Vascular Biology, 27, 487-493. doi:10.1161/01.ATV.0000255990.91805.6d

[11] Ferrari, G., Cook, B.D., Terushkin, V., Pintucci, G. and Mignatti, P. (2009) Transforming growth factor-beta 1 (TGF-beta1) induces angiogenesis through vascular endothelial growth factor (VEGF)-mediated apoptosis. Journal of Cellular Physiology, 219, 449-458. doi:10.1038/npre.2008.1758.1

[12] Thommen, R., Humar, R., Misevic, G., Pepper, M.S., Hahn, A.W., John, M. and Battegay, E.J. (1997) PDGF$\mathrm{BB}$ increases endothelial migration on cord movements during angiogenesis in vitro. Journal of Cellular Biochemistry, 64, 403-413.

doi:10.1002/(SICI)1097-4644(19970301)64:3<403::AIDJCB7>3.0.CO;2-Z

[13] Lange, S., Heger, J., Euler, G., Wartenberg, M., Piper, H.M. and Sauer, H. (2009) Platelet-Derived growth factor BB stimulates vasculogenesis of embryonic stem cell-derived endothelial cells by calcium-mediated generation of reactive oxygen species. Cardiovascular Research, 81, 159-168. doi:10.1093/cvr/cvn258

[14] Hao, X., Silva, E.A., Månsson-Broberg E.A., Grinnemo, K-H., Siddiqui, A.J., Dellgren, G., Wärdell E., Brodin, L.Å., Mooney, D.J. and Sylvén, C. (2007) Angiogenic effects of sequential release of VEGF-A165 and PDGF-BB with alginate hydrogels after myocardial infarction. Cardiovascular Research, 75, 178-185. doi:10.1016/j.cardiores.2007.03.028

[15] Stavridis, M.P., Collins, B.J. and Storey, K.G. (2010) Retinoic acid orchestrates fibroblast growth factor signalling to drive embryonic stem cell differentiation. Development, 137, 881-890. doi:10.1242/dev.043117

[16] Elizalde, C., Campa, V.M., Caro, M., Schlangen, K., Aransay, A.M., Del Mar Vivanco, M. and Kypta, R.M. (2010) Distinct roles for wnt-4 and wnt-11 during retinoic acid-induced neuronal differentiation. Stem Cells, 29, 141-153. doi: $10.1002 /$ stem.562

[17] Seifert, T., Stoelting, S., Wagner, T. and Peters, S.O. (2008) Vasculogeneic maturation of E14 embryonic stem cells with evidence of early vascular endothelial growth factor independency. Differentiation, 76, 857-867. doi:10.1111/j.1432-0436.2008.00271.x

[18] Kawamura, H., Li, X., Harper, S.J., Bates, D.O. and Claesson-Welsh, L. (2008) Vascular endothelial growth factor (VEGF)-A165b is a weak in vitro agonist for VEGF receptor-2 due to lack of coreceptor binding and deficient regulation of kinase activity. Cancer Research, 68, 4683-4692. doi:10.1158/0008-5472.CAN-07-6577

[19] Cao, R., Björndahl, M.A., Religa, P., Clasper, S., Garvin, S., Galter D., Meister, B., Ikomi, F., Tritsaris, K., Dissing, S., Ohhashi, T., Jackson, D.G., and Cao, Y. (2004) PDGF$\mathrm{BB}$ induces intratumoral lymphangiogenesis and promotes lymphatic metastasis. Cancer Cell, 6, 333-345. doi:10.1016/j.ccr.2004.08.034

[20] Yamashita, J.K. (2007) Differentiation of arterial, venous, and lymphatic endothelial cells from vascular progenitors. Trends in Cardiovascular Medicine, 17, 59-63. doi:10.1016/j.tcm.2007.01.001

[21] You, L.R., Lin, F.J., Lee, C.T., DeMayo, F.J., Tsai, M.J. and Tsai, S.Y. (2005) Suppression of Notch signalling by the COUP-TFII transcription factor regulates vein identity. Nature, 435, 98-104.

[22] Herbert, S.P., Huisken, J., Kim, T.N., Feldman, M.E., Houseman, B.T., Wang, R.A., Shokat, K.M. and Stainier, D.Y. (2009) Arterial-Venous segregation by selective cell sprouting: An alternative mode of blood vessel formation. 
Science, 326, 294-298. doi:10.1126/science.1178577

[23] Yamamizu, K., Matsunaga, T., Uosaki, H., Fukushima, H., Katayama, S., Hiraoka-Kanie, M., Mitani, K. and Yamashita, J.K. (2010) Convergence of Notch and beta-catenin signaling induces arterial fate in vascular progenitors. Journal of Cell Biology, 189, 325-338.

[24] Seki, T., Hong, K.H. and Oh, S.P. (2006) Nonoverlapping expression patterns of ALK1 and ALK5 reveal distinct roles of each receptor in vascular development. Laboratory Investigation, 86, 116-129. doi:10.1038/labinvest.3700376

[25] Kokudo, T., Suzuki, Y., Yoshimatsu, Y., Yamazaki, T., Watabe, T. and Miyazono, K. (2008) Snail is required for TGFbeta-induced endothelial-mesenchymal transition of embryonic stem cell-derived endothelial cells. Journal of Cell Science, 121, 3317-3324. doi:10.1242/jcs.028282 